\title{
Minimum Dissipation Theorem for Microswimmers
}

\author{
Babak Nasouri®, ${ }^{1}$ Andrej Vilfan $\odot,{ }^{1,2, *}$ and Ramin Golestanian $\circledast^{1,3}$ \\ ${ }^{1}$ Max Planck Institute for Dynamics and Self-Organization (MPIDS), 37077 Göttingen, Germany \\ ${ }^{2}$ Jožef Stefan Institute, 1000 Ljubljana, Slovenia \\ ${ }^{3}$ Rudolf Peierls Centre for Theoretical Physics, University of Oxford, Oxford OXI 3PU, United Kingdom
}

(Received 1 October 2020; accepted 23 December 2020; published 22 January 2021)

\begin{abstract}
We derive a theorem for the lower bound on the energy dissipation rate by a rigid surface-driven active microswimmer of arbitrary shape in a fluid at a low Reynolds number. We show that, for any swimmer, the minimum dissipation at a given velocity can be expressed in terms of the resistance tensors of two passive bodies of the same shape with a no-slip and perfect-slip boundary. To achieve the absolute minimum dissipation, the optimal swimmer needs a surface velocity profile that corresponds to the flow around the perfect-slip body, and a propulsive force density that corresponds to the no-slip body. Using this theorem, we propose an alternative definition of the energetic efficiency of microswimmers that, unlike the commonly used Lighthill efficiency, can never exceed unity. We validate the theory by calculating the efficiency limits of spheroidal swimmers.
\end{abstract}

DOI: 10.1103/PhysRevLett.126.034503

Microswimmers are natural or artificial self-propelled microscale objects moving through a fluid at low Reynolds numbers, such that viscous forces dominate over inertia [1]. The swimming motion can arise from periodic changes in the shape of the swimmer, which have to be nonreciprocal in time [2-4]. Organisms propelled by bacterial or eukaryotic flagella rely on nonreciprocal shape changes of their flagella. Many other microorganisms are propelled by thousands of cilia that all beat in an asymmetric fashion. Their beating could in principle be described as a shape change, but it is usually more insightful to use a coarsegrained approach in which the cilia are replaced by a propulsive layer that generates an effective tangential slip velocity along the surface [5-12]. Most artificial microswimmers rely on the self-phoretic mechanism and are therefore driven by a slip velocity by design [13-17].

The energetic efficiency of microswimmers is commonly defined by Lighthill's criterion as the power, needed when an external force moves a swimmer with drag coefficient $R$ with a speed $V$, divided by the dissipated power $P$ when the self-propelled swimmer moves with the same speed, namely $\eta_{L}=R V^{2} / P$ [5]. Maximizing $\eta_{L}$ will always provide the minimum power needed to achieve a certain swimming speed, or, conversely, the maximum speed that can be achieved with a given power. However, $\eta_{L}$ is not an

Published by the American Physical Society under the terms of the Creative Commons Attribution 4.0 International license. Further distribution of this work must maintain attribution to the author(s) and the published article's title, journal citation, and DOI. Open access publication funded by the Max Planck Society. efficiency in the thermodynamic sense and it can, in principle, exceed 100\% [18]. For instance, Leshansky et al. [19] showed that the Lighthill efficiency of a prolate spheroid diverges with the aspect ratio, becoming infinite for a thin needle. It is possible to introduce an efficiency that has an upper bound by evaluating the potential ability of the swimmer to tow a tethered cargo [18,20], although this definition will depend on the specific (geometric) features of the cargo [21].

Microswimmers driven by an effective surface slip velocity have two contributions to the dissipation: external (in the outer problem), due to the shearing motion of the surrounding fluid, and internal (in the inner problem), due to losses in the propulsive layer. The latter, which focuses on the dissipation in the propulsive layer [10], has been the focus of several studies on the grounds that this is often the dominant contribution, for example, in ciliated microorganisms [22,23]. The former, on the other hand, concerns the energy loss due to viscous dissipation, which has also been discussed analytically for spherical [24,25] and spheroidal [19] swimmers or computationally for more general axisymmetric swimmers [26]. Studies on phoretic swimmers also add the dissipation of the chemical mechanism that leads to the surface slip [27,28]. Here, our focus is on the external dissipation, which can set a fundamental limit on swimming efficiency, independent of the details of the driving mechanism. Since 1973, when John Blake proposed that minimum energy dissipation theorems be formulated [29], a number of analytical and numerical studies on efficiency limits of microswimmers have emerged. However, general statements on efficiency bounds have remained scarce.

In this Letter, we propose a theorem that sets a fundamental lower bound on the external dissipation $P_{A}$ 


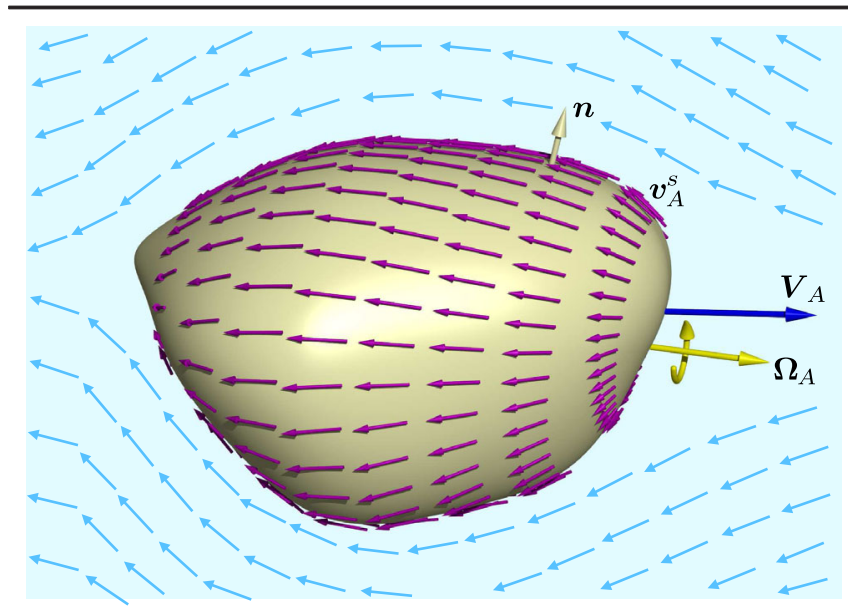

FIG. 1. A surface-slip driven swimmer of arbitrary shape with normal vector $\boldsymbol{n}$. The fluid at the boundary has a slip velocity $\boldsymbol{v}_{A}^{s}$ relative to the swimmer body. The swimmer moves with the rigidbody velocity $\mathbf{V}_{A}=\left[\boldsymbol{V}_{A} \boldsymbol{\Omega}_{A}\right]$, where $\boldsymbol{V}_{A}$ and $\boldsymbol{\Omega}_{A}$ are the translational and rotational velocities. The magenta and light-blue arrows show the schematic of the slip velocity and the streamlines, in the comoving frame.

around a surface-driven active microswimmer of any arbitrary shape with any swimming velocity (Fig. 1).

The swimmer moves with translational and angular velocities $\boldsymbol{V}_{A}$ and $\boldsymbol{\Omega}_{A}$ (or in short $\mathbf{V}_{A}=\left[\boldsymbol{V}_{A} \boldsymbol{\Omega}_{A}\right]$ ), which result from a surface slip velocity profile $\boldsymbol{v}_{A}^{s}$ that is always tangential to the surface (i.e., $\boldsymbol{v}_{A}^{s} \cdot \boldsymbol{n}=0$, where $\boldsymbol{n}$ is the surface normal). We prove that in the space of all possible configurations of $\boldsymbol{v}_{A}^{s}$ that lead to the same $\mathbf{V}_{A}$, the dissipation satisfies the inequality

$$
P_{A} \geq \mathbf{V}_{A} \cdot\left(\mathbf{R}_{\mathrm{PS}}^{-1}-\mathbf{R}_{\mathrm{NS}}^{-1}\right)^{-1} \cdot \mathbf{V}_{A}
$$

Here, $\mathbf{R}_{\mathrm{PS}}$ and $\mathbf{R}_{\mathrm{NS}}$ are the rigid-body resistance tensors corresponding to the perfect-slip (PS) and no-slip (NS), and they map the translational and angular velocities to the net hydrodynamic forces and torques, namely $\boldsymbol{F}_{\mathrm{PS}}=\left[\boldsymbol{F}_{\mathrm{PS}} \boldsymbol{L}_{\mathrm{PS}}\right]$ and $\mathbf{F}_{\mathrm{NS}}=\left[\boldsymbol{F}_{\mathrm{NS}} \boldsymbol{L}_{\mathrm{NS}}\right]$. We thus demonstrate that the minimum dissipation at a given swimming speed-or the maximum swimming efficiency — of an active swimmer can be determined solely by the knowledge of the viscous resistance tensors of the same body with no-slip and perfect-slip boundary conditions. We use the theorem to propose a new expression for the microswimmer efficiency as

$$
\eta_{m} \equiv \frac{\mathbf{V}_{A} \cdot \mathbf{R}_{\mathrm{PS}} \cdot \mathbf{V}_{A}}{P_{A}} \leq 1,
$$

which compares the absolute minimum power needed to move the body with the same exact velocity (corresponding to dragging a perfect-slip body) and the power expended by the active swimmer. We demonstrate that our proposed microswimmer efficiency is bounded by unity, unlike Lighthill efficiency.

We consider an active swimmer of arbitrary shape, shown in Fig. 1. The motion of the swimmer is governed by the Stokes equations $\boldsymbol{\nabla} \cdot \boldsymbol{\sigma}=\mathbf{0}$ and $\boldsymbol{\nabla} \cdot \boldsymbol{v}=0$ subject to boundary condition $\boldsymbol{v}(\boldsymbol{x} \in \mathcal{S})=\boldsymbol{V}_{A}+\boldsymbol{\Omega}_{A} \times \boldsymbol{x}+\boldsymbol{v}_{A}^{s}$. Here, $\boldsymbol{v}$ is the velocity field, $\boldsymbol{\sigma}=-p \boldsymbol{I}+2 \mu \boldsymbol{E}$ is the stress field, $p$ is the pressure field, $\boldsymbol{E}=\left(\boldsymbol{\nabla} \boldsymbol{v}+\boldsymbol{\nabla} \boldsymbol{v}^{\top}\right) / 2$ is the strain-rate tensor, $\mu$ is the fluid viscosity, $\mathcal{S}$ describes the surface of the particle, and $\boldsymbol{x}$ is the position vector.

The power dissipated due to any motion in a viscous fluid can be equivalently expressed either as a surface integral of the mechanical energy flux, $P=-\int_{\mathcal{S}} d S \boldsymbol{n} \cdot \boldsymbol{\sigma} \cdot \boldsymbol{v}$, or as a volume integral of the density of dissipated power, $P=2 \mu \int_{\mathcal{V}} d V \boldsymbol{E}: \boldsymbol{E}$. Here, $\mathcal{V}$ represents the volume of the surrounding fluid and the symbol : denotes a twofold contraction (i.e., $\boldsymbol{E}: \boldsymbol{E}=E_{i j} E_{j i}$ ). The equivalence between the two expressions follows from the divergence theorem [30,31]. In the absence of any external force or torque (i.e., $\int_{\mathcal{S}} d S \boldsymbol{f}_{A}=\mathbf{0}$ and $\int_{\mathcal{S}} d S \boldsymbol{x} \times \boldsymbol{f}_{A}=\mathbf{0}$ ), the power dissipated by the swimmer then follows

$$
P_{A}=-\int_{\mathcal{S}} d S f_{A} \cdot v_{A}^{S}
$$

where $\boldsymbol{f}_{A}=\boldsymbol{n} \cdot \boldsymbol{\sigma}_{A}$ is the traction. Here, our main goal is to find a slip velocity profile $\boldsymbol{v}_{A}^{s}$ that minimizes $P_{A}$ for a swimmer of arbitrary shape, while keeping the swimming velocity $\mathbf{V}_{A}$ constant.

We start our derivation by first showing that among all flows around a body of a given shape, the flow that satisfies the perfect-slip boundary condition on the surface has the minimal dissipation. For this purpose we adapt the standard derivation of the Helmholtz minimum dissipation theorem (see Guazzelli and Morris [31]), which states that the Stokes flow has the minimum dissipated power compared to any other flow that satisfies the same boundary condition on velocity. In our derivation, we compare the dissipation in the flow with the perfect-slip boundary with that in a flow with any other slip velocity. We consider the motion of a passive perfect-slip body with tangential slip velocity $\boldsymbol{v}_{\mathrm{PS}}^{s}$ and rigid-body motion $\mathbf{V}_{\mathrm{PS}}=\left[\boldsymbol{V}_{\mathrm{PS}} \boldsymbol{\Omega}_{\mathrm{PS}}\right]$. By definition, the tangential component of the traction in such a motion is zero, namely $\boldsymbol{f}_{\mathrm{PS}}^{\|}=(\boldsymbol{I}-\boldsymbol{n} \boldsymbol{n}) \cdot \boldsymbol{f}_{\mathrm{PS}}=\mathbf{0}$. Any tangential perturbation in the slip profile of this motion (denoted by ') then alters the viscous dissipated power by

$$
\begin{aligned}
\Delta P & =2 \mu \int_{\mathcal{V}} d V\left[\left(\boldsymbol{E}_{\mathrm{PS}}+\boldsymbol{E}^{\prime}\right):\left(\boldsymbol{E}_{\mathrm{PS}}+\boldsymbol{E}^{\prime}\right)-\boldsymbol{E}_{\mathrm{PS}}: \boldsymbol{E}_{\mathrm{PS}}\right], \\
& =2 \mu \int_{\mathcal{V}} d V \boldsymbol{E}^{\prime}: \boldsymbol{E}^{\prime}+4 \mu \int_{\mathcal{V}} d V \boldsymbol{E}^{\prime}: \boldsymbol{E}_{\mathrm{PS}}
\end{aligned}
$$

where $\Delta P$ is the change in the dissipation due to the perturbation in the slip profile and $\boldsymbol{E}^{\prime}$ is the strain-rate 
tensor for the perturbation flow. Since both $\boldsymbol{E}^{\prime}$ and $\boldsymbol{E}_{\mathrm{PS}}$ are traceless symmetric tensors, and $\boldsymbol{\nabla} \cdot \boldsymbol{v}^{\prime}=0$, we have $2 \mu \boldsymbol{E}^{\prime}: \boldsymbol{E}_{\mathrm{PS}}=\boldsymbol{\nabla} \boldsymbol{v}^{\prime}: \boldsymbol{\sigma}_{\mathrm{PS}}$, where $\boldsymbol{\sigma}_{\mathrm{PS}}=-p_{\mathrm{PS}} \boldsymbol{I}+2 \mu \boldsymbol{E}_{\mathrm{PS}}$ is the stress field for the perfect-slip flow and $p_{\mathrm{PS}}$ is the corresponding pressure field. Using $\boldsymbol{\nabla} \cdot \boldsymbol{\sigma}_{\mathrm{PS}}=\mathbf{0}$, one obtains $\boldsymbol{\nabla} \boldsymbol{v}^{\prime}: \boldsymbol{\sigma}_{\mathrm{PS}}=\boldsymbol{\nabla} \cdot\left(\boldsymbol{v}^{\prime} \cdot \boldsymbol{\sigma}_{\mathrm{PS}}\right)$. Thence, by once again using the divergence theorem, we find $4 \mu \int_{\mathcal{V}} d V \boldsymbol{E}^{\prime}: \boldsymbol{E}_{\mathrm{PS}}=$ $-2 \int_{\mathcal{S}} d S f_{\mathrm{PS}} \cdot \boldsymbol{v}^{\prime}=0$ (since $\boldsymbol{f}_{\mathrm{PS}}^{\|}=\mathbf{0}$ and $\boldsymbol{n} \cdot \boldsymbol{v}^{\prime}=0$ ). Now, noting that $2 \mu \int_{\mathcal{V}} d V \boldsymbol{E}^{\prime}: \boldsymbol{E}^{\prime}$ is positive definite, we can conclude that the minimum dissipated power can be only achieved when $\boldsymbol{E}^{\prime}=\mathbf{0}$, thereby indicating that the optimal slip velocity profile is that of a perfect-slip body. We can alternatively state that the dissipation of any motion with rigid-body velocity $\mathbf{V}_{\mathrm{PS}}$ is more than (or equal to) that of a perfect-slip body. For a perfect-slip body, the dissipated power is found $P_{\mathrm{PS}}=-\int_{\mathcal{S}} d S \boldsymbol{f}_{\mathrm{PS}} \cdot \boldsymbol{v}_{\mathrm{PS}}=\mathbf{V}_{\mathrm{PS}} \cdot \mathbf{R}_{\mathrm{PS}} \cdot \mathbf{V}_{\mathrm{PS}}$. Thus, we have proven

$$
P \geq \mathbf{V} \cdot \mathbf{R}_{\mathrm{PS}} \cdot \mathbf{V},
$$

where $P$ is the dissipated power in any flow with rigid-body motion $\mathbf{V}$. We note that there is an interesting difference between the implications of the Helmholtz theorem for no-slip and perfect-slip bodies. For no-slip bodies, the Helmholtz theorem implies that adding extra volume to the body, such that the old shape is fully contained in the new one, will always increase its drag coefficient [32]. This is because the flow around the enlarged body can be viewed as a non-optimal solution to the original problem. For perfect-slip bodies, however, no such statement is possible. For example, a perfect-slip spheroid reduces its drag coefficient upon elongation in the direction of motion while keeping the equatorial radius constant [33].

We now need to show how the inequality given in Eq. (5) sets an absolute lower bound for the dissipated power of an active swimmer of the same shape with velocity $\mathbf{V}_{A}$. Let us consider the motion of a body that is a linear superposition of an active swimmer with velocity $\mathbf{V}_{A}$ and a no-slip body of the same shape with velocity $\mathbf{V}_{\mathrm{NS}}$. From (5), we have a lower bound on the dissipated power in the superposition system

$$
P_{A+\mathrm{NS}} \geq\left(\mathbf{V}_{A}+\mathbf{V}_{\mathrm{NS}}\right) \cdot \mathbf{R}_{\mathrm{PS}} \cdot\left(\mathbf{V}_{A}+\mathbf{V}_{\mathrm{NS}}\right) .
$$

On the other hand, the dissipated power can be directly expressed as $P_{A+\mathrm{NS}}=-\int_{\mathcal{S}} d S\left(\boldsymbol{f}_{A}+\boldsymbol{f}_{\mathrm{NS}}\right) \cdot \boldsymbol{v}_{A+\mathrm{NS}}$, where $\boldsymbol{v}_{A+\mathrm{NS}}=\boldsymbol{v}_{A}^{S}+\boldsymbol{V}_{A}+\boldsymbol{V}_{\mathrm{NS}}+\left(\boldsymbol{\Omega}_{A}+\boldsymbol{\Omega}_{\mathrm{NS}}\right) \times \boldsymbol{x}$ at the boundary. This dissipated power then evaluates to

$P_{A+\mathrm{NS}}=-\int_{\mathcal{S}} d S\left(\boldsymbol{f}_{A}+\boldsymbol{f}_{\mathrm{NS}}\right) \cdot \boldsymbol{v}_{A}^{S}-\mathbf{F}_{\mathrm{NS}} \cdot\left(\mathbf{V}_{A}+\mathbf{V}_{\mathrm{NS}}\right)$.

Now we can employ the Lorentz reciprocal theorem to connect the active swimming problem to the no-slip one $[24,30,37-40]$. Since the flow field in the active problem is force- and torque-free, we find $\int_{\mathcal{S}} d S f_{\mathrm{NS}} \cdot \boldsymbol{v}_{A}^{s}=-\mathbf{F}_{\mathrm{NS}} \cdot \mathbf{V}_{A}$, which simplifies Eq. (7) to

$$
P_{A+\mathrm{NS}}=P_{A}-\mathbf{F}_{\mathrm{NS}} \cdot \mathbf{V}_{\mathrm{NS}},
$$

where $P_{A}$ and $-\mathbf{F}_{\mathrm{NS}} \cdot \mathbf{V}_{\mathrm{NS}}$ represent the dissipated power of the active and the no-slip particles, respectively. Thus, for the superposition of a force- and torque-free active particle and a no-slip body with arbitrary velocity, the dissipation rate is the sum of the two corresponding individual dissipation rate contributions.

The lower bound on the dissipation rate-given by Eqs. (6) and (8) - is valid for a superposition of an active swimmer with any slip profile $\boldsymbol{v}_{A}^{s}$ (resulting in the swimming velocity $\mathbf{V}_{A}$ ) and a no-slip body with any velocity $\mathbf{V}_{\mathrm{NS}}$. The equality (i.e., the minimum dissipation for the swimmer) is fulfilled if and only if the superposition represents a flow around a perfect-slip body. Among all $\mathbf{V}_{\mathrm{NS}}$, we therefore obtain the strictest bound on the dissipation when the velocities fulfill the conditions of the superposition described in Fig. 2(a). The velocities then must follow $\mathbf{V}_{\mathrm{PS}}=\mathbf{V}_{A}+\mathbf{V}_{\mathrm{NS}}$, and the force balance of the

(a)

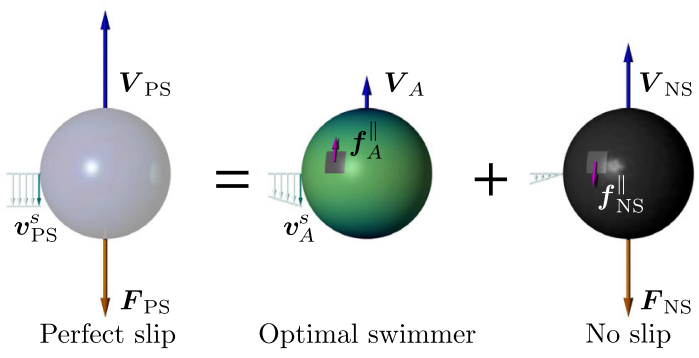

(b)

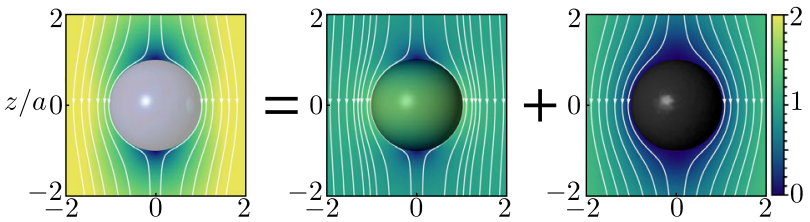

(c)

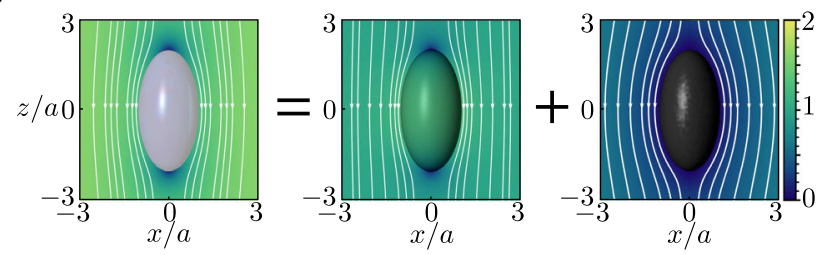

FIG. 2. (a) Schematic of the superposition. A perfect-slip body is represented as a superposition of the optimal active swimmer and a no-slip body. The cyan-colored arrows schematically show the velocity profile near the surface of the swimmer (in the comoving frame). Magenta-colored arrows show the tangential traction force density. This superposition is shown for two examples of (b) a sphere of radius $a$ and (c) a prolate spheroid with aspect ratio $b / a=2$. The white arrows show the streamlines and the colors indicate the value of the velocity scaled by the swimming speed of the active particle. All the figures are in the comoving Cartesian $x-z$ frame set at the center of the particle. 
superposition dictates $\mathbf{R}_{\mathrm{PS}} \cdot \mathbf{V}_{\mathrm{PS}}=\mathbf{R}_{\mathrm{NS}} \cdot \mathbf{V}_{\mathrm{NS}} \quad$ (since $\mathbf{F}_{\mathrm{PS}}=\mathbf{F}_{\mathrm{NS}}$ ). The velocities can then be expressed in terms of $\mathbf{V}_{A}$ as

$$
\begin{aligned}
& \mathbf{V}_{\mathrm{PS}}=\left(\mathbf{I}-\mathbf{R}_{\mathrm{NS}}^{-1} \cdot \mathbf{R}_{\mathrm{PS}}\right)^{-1} \cdot \mathbf{V}_{A}, \\
& \mathbf{V}_{\mathrm{NS}}=\left(\mathbf{R}_{\mathrm{PS}}^{-1} \cdot \mathbf{R}_{\mathrm{NS}}-\mathbf{I}\right)^{-1} \cdot \mathbf{V}_{A},
\end{aligned}
$$

where $\mathbf{I}$ is the six-dimensional identity tensor. Inserting these velocities in Eqs. (6) and (8) yields our main result that is presented in Eq. (1). Equation (1) shows that the minimum dissipated power by a swimmer can be obtained explicitly by evaluating the resistance tensors of two passive problems: the perfect-slip body and the no-slip body motion of the same geometry. This result is general and holds for any swimmer of any arbitrary shape (the simplified version for axisymmetric swimmers is presented in the Supplemental Material [33]). The optimization problem is remarkably reduced down to finding the resistance tensor of two passive systems in the same domain.

As discussed, the power dissipated in active swimming reaches its lower bound when the superposition of the active and no-slip cases conspires to exactly recreate the perfect-slip motion. Note that an unlimited variety of slip profiles can lead to the same swimming velocity, but only the superposition of the optimal swimmer and the no-slip body can amount to the flow field of a perfect-slip body. In other words, such a decomposition of the perfect-slip body uniquely demands $\boldsymbol{v}_{A}^{s}=\boldsymbol{v}_{\mathrm{PS}}^{s}$. From this, one can claim that the optimal slip velocity profile for any swimmer with swimming velocity $\mathbf{V}_{A}$ is identical to that of a perfect-slip body moving with a velocity given in Eq. (9). Furthermore, since for the perfect-slip body $\boldsymbol{f}_{\mathrm{PS}}^{\|}=\mathbf{0}$, the superposition also necessitates $f_{A}^{\|}=-f_{\mathrm{NS}}^{\|}$. Thus, we can similarly claim that the force density of an optimal swimmer is the negative of that induced by a passive motion of a no-slip body with the velocity given in (10).

The expression for the minimum power can also be used to find the maximum Lighthill efficiency. Recalling that the total power needed for dragging a no-slip body with velocity $\mathbf{V}_{A}$ is $\mathbf{V}_{A} \cdot \mathbf{R}_{\mathrm{NS}} \cdot \mathbf{V}_{A}$, we find

$$
\eta_{L} \leq \frac{\mathbf{V}_{A} \cdot \mathbf{R}_{\mathrm{NS}} \cdot \mathbf{V}_{A}}{\mathbf{v}_{A} \cdot\left(\mathbf{R}_{\mathrm{PS}}^{-1}-\mathbf{R}_{\mathrm{NS}}^{-1}\right)^{-1} \cdot \mathbf{V}_{A}}
$$

Expression (11) is also general and sets the upper bound for the efficiency of a swimmer with a given shape and swimming velocity. If the motion is axisymmetric with no rotation, the limit on efficiency takes the very simple form

$$
\eta_{L} \leq \frac{R_{\mathrm{NS}}}{R_{\mathrm{PS}}}-1
$$

where $R_{\mathrm{NS}}$ and $R_{\mathrm{PS}}$ are now the scalar drag coefficients of the no-slip and perfect-slip passive bodies. For instance, for a spherical swimmer (i.e., a squirmer [5,6,41]), we have $R_{\mathrm{NS}}=6 \pi \mu a$ and $R_{\mathrm{PS}}=4 \pi \mu a$, where $a$ is the radius [30]. This gives $\eta_{L}^{\max }=1 / 2$, a result which was also found by Michelin and Lauga [25]. From the known solutions for the flow around a bubble we know that the slip velocity of the optimal spherical swimmer is $v_{A}^{s}=\frac{3}{2} V_{A} \sin \theta$ with $\theta$ being the polar angle, also in agreement with Refs. [7,25]. The force density, which is identical to the force on a no-slip sphere translating with velocity $\left(R_{\mathrm{NS}} / R_{\mathrm{PS}}-1\right)^{-1} V_{A}$, is then simply found as $f_{A}^{\|}=3 \mu\left(V_{A} / a\right) \sin \theta$. The flow decomposition for an optimal spherical swimmer is shown in Fig. 2(b).

To highlight the strength of expression (12), we may also use it to evaluate the maximum efficiency of an axisymmetric spheroidal swimmer. The exact expression for the flow field and the drag coefficient of a no-slip spheroidal body is well documented [30], and we can similarly find the flow field and the drag coefficient for the perfect-slip body using an exact approach [33] [see Fig. 2(c) for an example]. By using these results, we can evaluate the maximum Lighthill efficiency for any value of the spheroid aspect ratio $b / a$. As shown in Fig. 3, our results precisely match the recent computational data for optimal swimming of spheroidal particles obtained with the boundary element method and numerical optimization by Guo et al. [26]. These results provide a fully independent validation of our theorem.

Our simple expression for the maximum efficiency also shows how the Lighthill efficiency diverges once the aspect ratio becomes increasingly large $b / a \rightarrow \infty$ (spheroid transforms to a needle) [19]. In that case, $R_{\mathrm{NS}} \propto b / \log (b / a) \rightarrow$ $\infty$ while $R_{\mathrm{PS}} \propto a^{2} / b \rightarrow 0$, resulting in $\eta_{L}^{\max } \propto(b / a)^{2} /$ $\log (b / a) \rightarrow \infty$. To resolve this, we propose an alternative microswimmer efficiency via Eq. (2), which yields

$$
\eta_{m} \leq \frac{\mathbf{V}_{A} \cdot \mathbf{R}_{\mathrm{PS}} \cdot \mathbf{V}_{A}}{\mathbf{V}_{A} \cdot\left(\mathbf{R}_{\mathrm{PS}}^{-1}-\mathbf{R}_{\mathrm{NS}}^{-1}\right)^{-1} \cdot \mathbf{V}_{A}},
$$

and consequently

$$
\eta_{m} \leq 1-\frac{R_{\mathrm{PS}}}{R_{\mathrm{NS}}}
$$

for axisymmetric bodies. Using this definition, for a spherical swimmer $\eta_{m}^{\max }=1 / 3$, and for a needle $\eta_{m}^{\max } \rightarrow 1$, see Fig. 3. We have demonstrated that the motion of a perfect-slip body has the minimum dissipation among all other types of motion, and therefore, unlike the Lighthill efficiency, $\eta_{m}$ is clearly bounded by unity.

In conclusion, we were able to express the minimum dissipation needed by an active swimmer using only two rigid-body resistance tensors of bodies with the same shape: a no-slip boundary condition in the first case and 


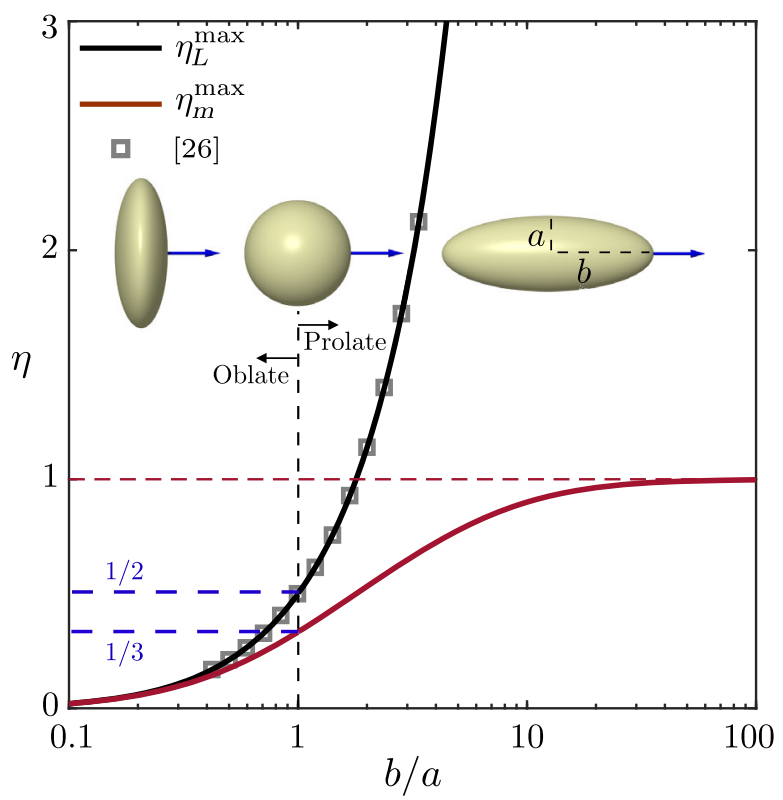

FIG. 3. Maximum Lighthill efficiency $\eta_{L}^{\max }$ (black line) of spheroidal swimmers as a function of the aspect ratio $b / a$, obtained using our theorem. The Lighthill efficiency diverges for prolate spheroids as $b / a$ increases. The red line shows our proposed microswimmer efficiency $\eta_{m}^{\max }$, which is bounded by unity. For a sphere $(b / a=1)$, our theorem recovers the welldocumented maximum Lighthill efficiency of $1 / 2$ [25], which corresponds to $1 / 3$ in the case of our proposed microswimmer efficiency. The grey squares show optimal efficiencies obtained by Guo et al. [26] using the boundary element method and numerical optimization.

a perfect-slip boundary in the second. We showed that the surface slip velocities and forces that reach this minimal dissipation correspond to the flow around a perfect-slip body and the tangential force on the no-slip body. We have thus reduced a complex optimization problem to the calculation of two resistance tensors, for which numerous analytical solutions and numerical methods are available.

In this study, we found a general theorem on the minimum dissipation of surface-driven microswimmers. An outstanding challenge will be to generalize this theorem to swimmers that propel themselves by changing their shapes $[3,42,43]$.

We thank Evelyn Tang for comments on the manuscript. This work has been supported by the Max Planck Society. A. V. acknowledges support from the Slovenian Research Agency (Grant No. P1-0099).

*andrej.vilfan@ds.mpg.de

[1] G. Gompper et al., The 2020 motile active matter roadmap, J. Phys. Condens. Matter 32, 193001 (2020).

[2] E. M. Purcell, Life at low Reynolds number, Am. J. Phys. 45, 3 (1977).
[3] A. Najafi and R. Golestanian, Simple swimmer at low Reynolds number: Three linked spheres, Phys. Rev. E 69, 062901 (2004).

[4] B. Nasouri, A. Vilfan, and R. Golestanian, Efficiency limits of the three-sphere swimmer, Phys. Rev. Fluids 4, 073101 (2019).

[5] M. J. Lighthill, On the squirming motion of nearly spherical deformable bodies through liquids at very small Reynolds numbers, Commun. Pure Appl. Math. 5, 109 (1952).

[6] J. R. Blake, A spherical envelope approach to ciliary propulsion, J. Fluid Mech. 46, 199 (1971).

[7] J. Blake, A finite model for ciliated micro-organisms, J. Biomech. 6, 133 (1973).

[8] F. Jülicher and J. Prost, Generic theory of colloidal transport, Eur. Phys. J. E 29, 27 (2009).

[9] N. Osterman and A. Vilfan, Finding the ciliary beating pattern with optimal efficiency, Proc. Natl. Acad. Sci. U.S.A. 108, 15727 (2011).

[10] A. Vilfan, Optimal Shapes of Surface Slip Driven SelfPropelled Microswimmers, Phys. Rev. Lett. 109, 128105 (2012).

[11] T. J. Pedley, D. R. Brumley, and R. E. Goldstein, Squirmers with swirl: A model for Volvox swimming, J. Fluid Mech. 798, 165 (2016).

[12] A. W. Zantop and H. Stark, Squirmer rods as elongated microswimmers: Flow fields and confinement, Soft Matter 16, 6400 (2020).

[13] J. L. Anderson and D. C. Prieve, Diffusiophoresis caused by gradients of strongly adsorbing solutes, Langmuir 7, 403 (1991).

[14] R. Golestanian, T. B. Liverpool, and A. Ajdari, Propulsion of a Molecular Machine by Asymmetric Distribution of Reaction Products, Phys. Rev. Lett. 94, 220801 (2005).

[15] A. I. Campbell, S. J. Ebbens, P. Illien, and R. Golestanian, Experimental observation of flow fields around active Janus spheres, Nat. Commun. 10, 3952 (2019).

[16] B. Nasouri and R. Golestanian, Exact Phoretic Interaction of Two Chemically Active Particles, Phys. Rev. Lett. 124, 168003 (2020).

[17] R. Pöhnl, M. N. Popescu, and W. E. Uspal, Axisymmetric spheroidal squirmers and self-diffusiophoretic particles, J. Phys. Condens. Matter 32, 164001 (2020).

[18] S. Childress, A thermodynamic efficiency for Stokesian swimming, J. Fluid Mech. 705, 77 (2012).

[19] A. M. Leshansky, O. Kenneth, O. Gat, and J. E. Avron, A frictionless microswimmer, New J. Phys. 9, 145 (2007).

[20] O. Raz and A. M. Leshansky, Efficiency of cargo towing by a microswimmer, Phys. Rev. E 77, 055305(R) (2008).

[21] R. Golestanian, Three-sphere low-Reynolds-number swimmer with a cargo container, Eur. Phys. J. E 25, 1 (2008).

[22] S. R. Keller and T. Y. Wu, A porous prolate-spheroidal model for ciliated micro-organisms, J. Fluid Mech. 80, 259 (1977).

[23] H. Ito, T. Omori, and T. Ishikawa, Swimming mediated by ciliary beating: Comparison with a squirmer model, J. Fluid Mech. 874, 774 (2019).

[24] H. A. Stone and A. D. T. Samuel, Propulsion of Microorganisms by Surface Distortions, Phys. Rev. Lett. 77, 4102 (1996). 
[25] S. Michelin and E. Lauga, Efficiency optimization and symmetry-breaking in a model of ciliary locomotion, Phys. Fluids 22, 111901 (2010).

[26] H. Guo, H. Zhu, R. Liu, M. Bonnet, and S. Veerapaneni, Optimal slip velocities of micro-swimmers with arbitrary axisymmetric shapes, J. Fluid Mech. 910, A26 (2021).

[27] B. Sabass and U. Seifert, Efficiency of Surface-Driven Motion: Nanoswimmers Beat Microswimmers, Phys. Rev. Lett. 105, 218103 (2010).

[28] B. Sabass and U. Seifert, Dynamics and efficiency of a selfpropelled, diffusiophoretic swimmer, J. Chem. Phys. 136, 064508 (2012).

[29] John Blake predicted that "The problem of maximizing the velocity of propulsion while keeping the rate of energy dissipation fixed is likely to lead to interesting minimum energy dissipation theorems" [7].

[30] J. Happel and H. Brenner, Low Reynolds Number Hydrodynamics (Springer Netherlands, Dordrecht, 1983).

[31] E. Guazzelli and J. F. Morris, A Physical Introduction to Suspension Dynamics (Cambridge University Press, Cambridge, England, 2009).

[32] R. Hill and G. Power, Extremum principles for slow viscous flow and the approximate calculation of drag, Q. J. Mech. Appl. Math. 9, 313 (1956).

[33] See the Supplemental Material at http://link.aps.org/ supplemental/10.1103/PhysRevLett.126.034503 for the summary of the theorem for axisymmetric swimmers and the details of the calculations for the motion of a perfect-slip spheroidal particle, which includes Refs. [34-36].
[34] G. Dassios, M. Hadjinicolaou, and A.C. Payatakes, Generalized eigenfunctions and complete semiseparable solutions for Stokes flow in spheroidal coordinates, Q. Appl. Math. 52, 157 (1994).

[35] S. Deo and S. Datta, Stokes flow past a fluid prolate spheroid, Indian J. Pure Appl. Math. 34, 755 (2003).

[36] L. E. Payne and W. H. Pell, The Stokes flow problem for a class of axially symmetric bodies, J. Fluid Mech. 7, 529 (1960).

[37] H. A. Lorentz, Eene algemeene stelling omtrent de beweging eener vloeistof met wrijving en eenige daaruit afgeleide gevolgen, Zittingsverslag K. Akad. Wet. Amsterdam 5, 168 (1896).

[38] G. J. Elfring, Force moments of an active particle in a complex fluid, J. Fluid Mech. 829, R3 (2017).

[39] B. Nasouri and G. J. Elfring, Higher-order force moments of active particles, Phys. Rev. Fluids 3, 044101 (2018).

[40] H. Masoud and H. A. Stone, The reciprocal theorem in fluid dynamics and transport phenomena, J. Fluid Mech. 879, P1 (2019).

[41] O. S. Pak and E. Lauga, Generalized squirming motion of a sphere, J. Eng. Math. 88, 1 (2014).

[42] M. A. Jalali, M.-R. Alam, and S. H. Mousavi, Versatile lowReynolds-number swimmer with three-dimensional maneuverability, Phys. Rev. E 90, 053006 (2014).

[43] M. Mirzakhanloo, M. A. Jalali, and M.-R. Alam, Hydrodynamic choreographies of microswimmers, Sci. Rep. 8, 3670 (2018). 